\title{
Partial Blockage
}

National Cancer Institute

\section{Source}

National Cancer Institute. Partial Blockage. NCI Thesaurus. Code C63289.

Problem related to an obstruction or blockage within the device component (e.g. tube, opening, pipe) that results in a reduction of the flow rate. 